\title{
Flight Dynamics and Stability of Kites in Steady and Unsteady Wind Conditions
}

\author{
L. Salord Losantos ${ }^{*}$ and G. Sánchez-Arriaga ${ }^{\dagger}$
}

\begin{abstract}
The flight dynamics and stability of a kite with a single main line flying in steady and unsteady wind conditions are discussed. A simple dynamic model with five degrees of freedom is derived with the aid of Lagrangian formulation, which explicitly avoids any constraint force in the equations of motion. The longitudinal and latera-directional modes and stability of the steady flight under constant wind conditions are analyzed by using both numerical and analytical methods. Taking advantage of the appearance of small dimensionless parameters in the model, useful analytical formulas for stable-designed kites are found. Under nonsteady wind-velocity conditions, the eguilibrium state disappears and periodic orbits occur. The kite stability and an interesting resonance phenomenon are explored with the aid of a numerical method based on Floquet theory.
\end{abstract}

\section{Nomenclature}

$\begin{array}{ll}A & =\text { kite surface, } \mathrm{m}^{2} \\ b & =\text { kite span, } \mathrm{m} \\ C_{l p}, C_{n r} & =\text { aerodynamic-torque coefficients, } \mathrm{s} / \mathrm{rad} \\ C_{t \beta}, C_{n \beta} & =\text { aerodynamic-torque coefficients } \\ C_{Z \alpha}, C_{Y \beta} & =\text { aerodynamic-force coefficients } \\ c & =\text { kite chord, } \mathrm{m} \\ F & =\text { aerodynamic force, } \mathrm{N} \\ g & =\text { gravitational acceleration, } \mathrm{m} / \mathrm{s}^{2} \\ L & =\text { tether length, } \mathrm{m} \\ l & =\text { bridle length, } \mathrm{m} \\ M & =\text { kite mass, } \mathrm{kg} \\ p, q, r & =\text { angular-velocity components, rad } / \mathrm{s} \\ r_{x, y,-} & =\text { radius of gyration, } \mathrm{m} \\ T & =\text { aerodynamic torque, } \mathrm{N} \cdot \mathrm{m} \\ V_{w} & =\text { wind speed, } \mathrm{m} / \mathrm{s} \\ \tilde{x}_{0}, \tilde{x}_{\alpha} & =\text { center-of-pressure coefficients } \\ \delta & =\text { bridle angle, } \mathrm{rad} \\ \rho_{0} & =\text { air density, } \mathrm{kg} / \mathrm{m}^{3}\end{array}$

\section{Introduction}

W IND-ENERGY and thrust-generation concepts based on kites have recently gained the interest of research groups and companies [1]. Two examples are the traction system developed by SkySails to reduce the fuel costs of ships, and the power generator by KiteGen [2]. The main advantage is the operational altitude that is of the order of thousands of meters (unlike the few hundreds of meters reached by conventional wind turbines). At such high altitudes, the energy density is much higher due to the strong and steady wind.

Pioneer wind-power-generation models like the crosswind kite power [3] or the Laddermill [4] have been followed by a single kite operating in a pumping mode [5]. This last concept has to face with the stability and control of the kite; in addition to the dynamic instabilities of towed bodies [6-8], one may also consider the changing wind conditions, the complex nature of the periodic trajectory, and the need of maximizing the energy production per cycle. Both the longitudinal [9] and lateral [10] stabilities of a rigid-solid kite have

Received 24 January 2014: revision received 29 August 2014; accepted for publication 30 August 2014; published online 9 December 2014. Copyright (C) 2014 by the American Institute of Aeronautics and Astronautics, Inc. All rights reserved. Copies of this paper may be made for personal or internal use. on condition that the copier pay the $\$ 10.00$ per-copy fee to the Copyright Clearance Center, Inc., 222 Rosewood Drive, Danvers, MA 01923: include the code 1533-3868/14 and $\$ 10.00$ in correspondence with the $\mathrm{CCC}$.

*Aerospace Engineer. Departamento de Física Aplicada, Escuela Técnica Superior de Ingenieros Aeronánticos.

${ }^{\dagger}$ Assistant Professor. Departamento de Física Aplicada, Escuela Técnica Superior de Ingenieros Aeronánticos. been also considered, as well as the bridle configuration [11] and the main-line design [12]. Recent works have included flexibility effects [13] and different control schemes [14-16].

The recent development on kite technology and flight simulators may be combined with simple models because they can provide a deeper understanding on the physics, including simple analytical formulas, relating the kite stability with the physical parameters (like bridle geometry, aerodynamic coefficient, and kite inertial properties). This was the goal of the present work, which extends the model introduced in [9] to include kite lateral dynamics and nonsteady wind conditions. It considers a solid rigid, made of the kite and the bridle, joined at a point $Q$ with a rigid massless rod (see Fig. 1). Both the aerodynamic drag on the rod and the friction at $Q$ and at the ground attachment point are ignored. As shown in [9], a Lagrangian formulation is very effective because the constraint forces (line tension at $Q$ ) do not appear explicitly in the equation of motion. Here. the length of the bridle lines is kept fixed in time, an issue that will be explored in a forthcoming work.

The paper is organized as follows. Section II introduces the model and the equations of motion of the kite. In Sec. III, we study the dynamics and stability of the kite under steady wind conditions, including its equilibrium state and the longitudinal and lateral stabilities. If the wind velocity varies periodically in time, then the equilibrium state is destroyed and periodic orbits appear. Their stability properties and a resonance phenomenon are explored in Sec. IV. Conclusions are summarized in Sec. V. For convenience, auxiliary operations have been included in the Appendix.

\section{The Model}

A. Kinematics and Forces

We used an Earth-fixed inertial frame with origin at the ground attachment point, $z_{E}$ axis along the vertical, wind velocity $\boldsymbol{w}_{0}$ contained in the $x_{E}-z_{E}$ plane, and $y_{E}$ axis forming a right-handed frame. For convenience, the trigonometric functions $\sin \alpha$ and $\cos \alpha$ of an angle $\alpha$ will be denoted by $s \alpha$ and $c \alpha$. Two angles, $\Gamma$ and $\varphi$. define the coordinates of point $Q$, where the main line of length $L$ and the bridle are joined. Vector $O Q$ is

$$
\boldsymbol{O Q}=-L\left(c \Gamma c \varphi i_{E}+c \Gamma s \varphi j_{E}+s \Gamma k_{E}\right)
$$

A body frame with origin at the center of mass $G$ of the kite and ax is equal to the principal axis of inertial relative to $G$ is also used. The tensor of inertia of the kite in this frame takes the form

$$
\boldsymbol{I}_{G}=m\left(\begin{array}{ccc}
r_{x}^{2} & 0 & 0 \\
0 & r_{y}^{2} & 0 \\
0 & 0 & r_{z}^{2}
\end{array}\right)
$$

in which $r_{x, y, z}$ are the radij of gyration. The orientation of the body frame with respect to the Earth frame is determined by Euler angles $\theta$. 


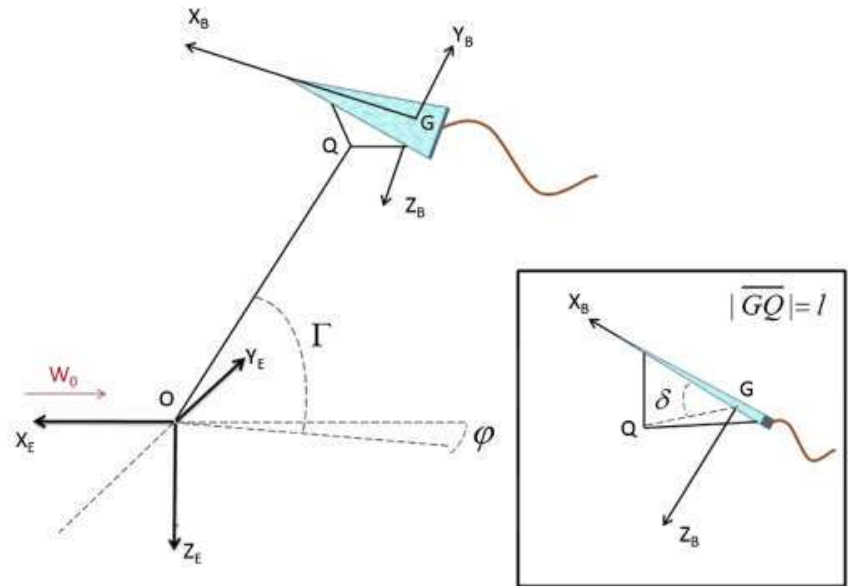

Fig. 1 Reference frames and coordinates of the model. Inset: bridle geometry.

$\psi$, and $\phi$, which correspond to the pitch, yaw, and roll angles of the kite, respectively. The dynamic state of the kite in our model is then given by the five angles

$$
\boldsymbol{x}=\left[\begin{array}{lllll}
\Gamma & \theta & \varphi & \psi & \phi
\end{array}\right]^{T}
$$

Euler angles are helpful because vector components in the Earth and body frames are related by the rotation matrix $\boldsymbol{R}$ given in Eq. (A1) of the Appendix, and the angular velocity $\omega$ of the kite is

$$
\omega=S^{(\omega)} \dot{x}
$$

in which the matrix $S^{(\omega)}$ depends on the state vector $x$ [see Eq. (A2)].

Following [9], we assume a rigid symmetric bridle with lines of fixed length. Bridle geometry is controlled by the $l$ and $\delta$ (see inset in Fig. 1), and we have $\boldsymbol{G} \boldsymbol{Q}=l\left(c \delta \boldsymbol{i}_{B}+s \delta \boldsymbol{k}_{B}\right)$. Using Eq. (A1), the center-of-mass position vector $\boldsymbol{r}_{G} \equiv \boldsymbol{O Q}+\boldsymbol{Q G}$ is

$$
\begin{aligned}
\boldsymbol{r}_{G} & =-L\left\{\left[c \Gamma c \varphi+\epsilon_{l}\left(c \psi f_{1}+s \delta s \psi s \phi\right)\right] i_{E}\right. \\
& \left.+\left[c \Gamma s \varphi+\epsilon_{l}\left(s \psi f_{1}-s \delta c \psi s \phi\right)\right] j_{E}+\left(s \Gamma-\epsilon_{l} f_{2}\right) k_{E}\right\}
\end{aligned}
$$

in which $f_{1} \equiv c \delta c \theta+s \delta s \theta c \phi$ and $f_{2} \equiv c \delta s \theta-s \delta c \theta c \phi$ are two auxiliary functions, and $\epsilon_{l} \equiv l / L$. The velocity $\boldsymbol{V}_{G}=\mathrm{d} \boldsymbol{r}_{G} / \mathrm{d} t$ of the center of mass can be found from Eq. (5). It reads

$$
V_{G}=L \boldsymbol{S}^{(v)} \dot{x}
$$

with $\boldsymbol{S}^{(v)}$ a matrix that just depends on the state vector $\boldsymbol{x}$ [see Eq. (A3)].

Both aerodynamic and gravitational forces act on the kite. The latter derives from the potential

$$
U(\boldsymbol{x}) \equiv M g L \tilde{U}=M g L\left[s \Gamma+\epsilon_{l}(s \delta c \theta c \phi-c \delta s \theta)\right]
$$

with $M$ the mass of the kite. The resultant $\boldsymbol{F}=\rho_{0} A V_{A}^{2} \tilde{\boldsymbol{F}} / 2$ and the torque about the center of mass $T=\rho_{0} A L V_{A}^{2} T_{M} / 2$ of the aerodynamic force are here calculated with the following simple model (valid for attack angles $\alpha$ below the stall):

$$
\begin{gathered}
\tilde{\boldsymbol{F}}=C_{Y \beta} \beta \boldsymbol{j}_{B}+C_{Z \alpha} \alpha \boldsymbol{k}_{B} \\
\tilde{\boldsymbol{T}}=\epsilon_{b}\left(C_{l \beta} \beta+C_{l \tilde{p}} \tilde{p}\right) \boldsymbol{i}_{B}-\epsilon_{c}\left(\tilde{x}_{0}-\tilde{x}_{\alpha} \alpha\right) C_{Z \alpha} \alpha j_{B} \\
+\epsilon_{b}\left(C_{n \beta} \beta+C_{n \tilde{r}} \tilde{r}\right) \boldsymbol{k}_{B}
\end{gathered}
$$

in which we introduced the dimensionless angular-velocity components in the body frame $\omega \equiv \sqrt{g / L}\left(\tilde{p} i_{B}+\tilde{q} j_{B}+\tilde{r} k_{B}\right)$. We remark that parameters $\tilde{x}_{0}$ and $\tilde{x}_{\alpha}$ control the relative position between the center of mass and the center of pressure of the kite (see [9]).

From the aerodynamic velocity $V_{A}=V_{G}+W_{0} i_{T}$, one finds the attack and sideslip angles:

$$
\alpha=\arctan \left(\frac{\boldsymbol{V}_{A} \cdot \boldsymbol{k}_{B}}{\boldsymbol{V}_{A} \cdot \boldsymbol{i}_{B}}\right), \quad \beta=\arcsin \left(\frac{\boldsymbol{V}_{A} \cdot \boldsymbol{j}_{B}}{\left|\boldsymbol{V}_{A}\right|}\right)
$$

The components of the aerodynamic velocity in the Earth frame are then given by

$$
V_{A i} \equiv \sqrt{g L} \tilde{V}_{A i}=\sqrt{g L}\left[S_{i k}^{(v)} \dot{x}_{k}+\tilde{V}_{w} \delta_{x, i}\right], \quad i=x, y, z
$$

in which $\tilde{V}_{w} \equiv W_{0} / \sqrt{L g}, \delta_{i j}$ is the Kronecker delta, and we used Einstein summation convention. Here, we assume the following (normalized) wind velocity:

$$
\tilde{V}_{w}=\tilde{V}_{0}\left[1+\tilde{V}_{1} \cos \Omega t\right]
$$

In Sec. III, we take $\tilde{V}_{1}=0$ (constant wind velocity) and, in Sec. IV, we fix $\tilde{V}_{1}=0.15$ and use $\Omega$ as a bifurcation parameter (steady wind conditions with a sinusoidal perturbation).

\section{B. Lagrange Equations}

Lagrange formulation provides a compact method to find the equations of motion of the kite. The constraint forces (tensions at point $Q$ ) do not appear explicitly in the equations because they do no work. The result is a second-order set of ordinary differential equations describing the evolution of the state vector $x$. Lagrange equations read

$$
\frac{\mathrm{d}}{\mathrm{d} t}\left(\frac{\partial \mathcal{L}}{\partial \dot{x}_{i}}\right)-\frac{\partial \mathcal{L}}{\partial x_{i}}=Q_{i}, \quad i=1, \ldots 5
$$

in which

$$
\mathcal{L}=\frac{1}{2} M V_{G}^{2}+\frac{1}{2} \boldsymbol{\omega} \cdot \boldsymbol{I}_{G} \cdot \boldsymbol{\omega}-U
$$

$\left(\partial \omega / \partial \dot{x}_{i}\right)$ is the generalized force of the coordinate $i$.

For convenience, from now on, we will use the dimensionless time $\tau \equiv t \sqrt{g / L}$ and the notation $\dot{x}=\mathrm{d} \boldsymbol{x} / \mathrm{d} \tau$. The equations of motion read

$$
\begin{aligned}
& M_{i j} \ddot{x}_{j}+\frac{\partial M_{i j}}{\partial x_{k}} \dot{x}_{j} \dot{x}_{k}-\frac{1}{2} \frac{\partial M_{j k}}{\partial x_{i}} \dot{x}_{j} \dot{x}_{k}+\frac{\partial \tilde{U}}{\partial x_{i}} \\
& =\mu \tilde{V}_{A j} \tilde{V}_{A j}\left[\tilde{F}_{k} R_{k m} S_{m i}^{(v)}+\varepsilon_{c} \tilde{T}_{k} S_{k i}^{(\omega)}\right] \quad i=1, \ldots 5
\end{aligned}
$$

in which $\boldsymbol{M}(\boldsymbol{x}) \equiv \boldsymbol{M}_{L}+\boldsymbol{M}_{R}$ and $\boldsymbol{M}_{L}$ and $\boldsymbol{M}_{\boldsymbol{R}}$ are given in Eqs. (A4) and (A5). Matrix $M$, which depends on the state vector $\boldsymbol{x}$ but not on $\dot{\boldsymbol{x}}$, contains information about the kinematics of the model. A timedependent bridle geometry or main-line length could be easily added to Eq. (14) because it only affects to $M$. On the other hand, the term $\partial \tilde{U} / \partial x_{i}$ and the right-hand side of Eq. (14) take into account the gravitational and aerodynamic forces, respectively.

In the analysis, we will use the numerical values given in Table 1, which correspond to a small kite. The angle $\delta$, the wind velocity $W_{0}$, and $\tilde{\Omega} \equiv \Omega \sqrt{L / g}$ will be used as control parameters. From Table 1 , one finds the following dimensionless values: $\epsilon_{l} \equiv l / L=0.01$, $\epsilon_{c} \equiv c / L=0.008, \epsilon_{x, y, z} \equiv r_{x, y, z} / L=(0.002,0.0025,0.0045), \mu \equiv$ $\rho_{0} A L / 2 M=98, C_{Y \beta}=-0.168, C_{Z \alpha}=-2.636, C_{l \beta}=-0.038$, $C_{l \tilde{p}}=-0.0292, C_{n \beta}=0.036, C_{n \tilde{r}}=-0.0071, \tilde{x}_{0}=0.3$, and $\tilde{x}_{\alpha}=$ 0.458 . For later calculations, we now introduce the state vector $\boldsymbol{u}=\left[\begin{array}{ll}\boldsymbol{x} & \dot{\boldsymbol{x}}\end{array}\right]$, and write system (13) as

$$
\frac{\mathrm{d} u}{\mathrm{~d} \tau}=f(\boldsymbol{u}, \tau)
$$


Table 1 Parameter values

\begin{tabular}{|c|c|c|c|}
\hline \multicolumn{2}{|c|}{ Physical parameters } & \multicolumn{2}{|c|}{ Dimensionless parameters } \\
\hline Symbol & Value & Symbol & Value \\
\hline$A$ & $0.48 \mathrm{~m}^{2}$ & $\overline{\tilde{x}_{0}}$ & 0.3 \\
\hline$c$ & $0.8 \mathrm{~m}$ & $\tilde{x}_{\alpha}$ & 0.458 \\
\hline$b$ & $0.6 \mathrm{~m}$ & $C_{s \alpha}$ & -2.636 \\
\hline$M$ & $0.3 \mathrm{~kg}$ & $C_{Y \beta}$ & -0.168 \\
\hline$r_{x}$ & $0.2 \mathrm{~m}$ & $C_{l \beta}$ & -0.038 \\
\hline$r_{y}$ & $0.25 \mathrm{~m}$ & $C_{l p}$ & $-0.0932 \mathrm{~s} / \mathrm{rad}$ \\
\hline$r_{2}$ & $0.45 \mathrm{~m}$ & $C_{n \beta}$ & 0.036 \\
\hline$l$ & $1 \mathrm{~m}$ & $C_{n r}$ & $-0.0228 \mathrm{~s} / \mathrm{rad}$ \\
\hline$L$ & $100 \mathrm{~m}$ & $\vec{V}_{1}$ & 0.15 \\
\hline$\rho_{0}$ & $1.225 \mathrm{~kg} / \mathrm{m}^{3}$ & & \\
\hline
\end{tabular}

a The wind velocity $W_{0}$ and the bridle angle $\delta$ are used as control parameters.

\section{Kite Stability Under Steady Wind Conditions}

\section{A. Perturbed Equations About the Equilibria}

In steady wind-velocity conditions $\left[\tilde{V}_{1}=0\right.$ in Eq. (12)], equilibrium states of the kite exist. In this case, system (15) is autonomous, and the equilibrium states $x^{*}$ are given by $f\left(u^{*}\right)=0$. Here, we restrict the analysis to symmetrical equilibrium solutions given by

$$
\boldsymbol{x}^{*}=\left[\begin{array}{lllll}
\Gamma^{*} & \theta^{*} & 0 & 0 & 0
\end{array}\right], \quad \dot{x}^{*}=0
$$

The conditions $f\left(x^{*}\right)=0$ yield the following pair of equations [9]:

$$
\begin{gathered}
\cos \left(\delta-\theta^{*}\right)+\sigma \theta^{*}\left[\frac{\epsilon_{c}}{\epsilon_{l}}\left(\tilde{x}_{0}-\tilde{x}_{\alpha} \theta^{*}\right)-\cos \delta\right]=0 \\
\Gamma^{*}=\arctan \left(\frac{\sigma \theta^{*} \cos \theta^{*}-1}{\sigma \theta^{*} \sin \theta^{*}}\right)
\end{gathered}
$$

with $\sigma \equiv-\mu \tilde{V}_{0}^{2} C_{Z \alpha}$. Equation (17), which is nonlinear and admits multiple solutions for exactly the same values of the parameters [9], gives the equilibrium pitch angle $\theta^{*}$. Once $\theta^{*}$ is known, one finds the elevation angle of the main line $\Gamma^{*}$ with Eq. (18). From now on, just the equilibrium state below the stall angle will be considered $(\alpha<25 \mathrm{deg})$.

The stability of the equilibrium states is analyzed by writing the state vector as $x=x^{*}+x^{1}$, in which $x^{1}$ is a small perturbation. Substituting this expansion in system (14) and ignoring terms of order $O\left(\left|x^{1}\right|^{2}\right)$ yield

$$
M^{*} \ddot{\boldsymbol{x}}^{1}+D^{*} \dot{\boldsymbol{x}}^{1}+K^{*} \boldsymbol{x}^{1}=0
$$

Matrices $M^{*}, D^{*}, K^{*}$, which are all evaluated at the equilibrium position, have a block structure that does not couple the longitudinal $\boldsymbol{x}_{L}^{1} \equiv\left[\begin{array}{ll}\Gamma^{1} & \theta^{1}\end{array}\right]$ and lateral-directional $\boldsymbol{x}_{D}^{1} \equiv\left[\begin{array}{lll}\varphi^{1} & \psi^{1} & \phi^{1}\end{array}\right]$ variables, thus allowing a separate analysis of the kite dynamics.

\section{B. Longitudinal Stability}

The longitudinal perturbations $x_{L}^{1} \equiv\left[\begin{array}{ll}\Gamma^{1} & \theta^{1}\end{array}\right]$ about the equilibrium position are described by

$$
M_{L}^{*} \ddot{x}_{L}^{1}+D_{L}^{*} \dot{x}_{L}^{1}+K_{L}^{*} x_{L}^{1}=0
$$

in which $M_{L}^{*}, D_{L}^{*}$, and $K_{L}^{*}$ are given by Eqs. (A6-A8). Because all these matrices are evaluated at the fixed point, they have constant coefficients. Taking solutions of the form $x_{L}^{1}(\tau)=x_{L 0} e^{\lambda \tau}$ in Eq. (20) yields a homogeneous set of algebraic equations. Its compatibility condition is

$$
a_{L} \lambda^{4}+b_{L} \lambda^{3}+c_{L} \lambda^{2}+d_{L} \lambda+e_{L}=0
$$

in which the coefficients $a_{L}-e_{L}$, which can be derived by using Eqs. (A6-A8), are intricate functions of the physical parameters. For instance, one finds $a_{L}=\epsilon_{y}^{2}+\epsilon_{l}^{2} \sin ^{2}(\delta-\Gamma-\theta)>0$.
In [9], Eq. (21) was investigated numerically. It was shown that, for parameters given in Table 1, it has two pairs of complex conjugated solutions. They are the pitch and pendular modes. Here, we exploit the disparate scales appearing in the model to derive some analytical results. Because one has $\epsilon_{l} \sim \epsilon_{c} \sim \epsilon_{x, y, z} \ll 1$, coefficients $a_{L}-e_{L}$ have different orders of magnitudes, and the four solutions of Eq. (21) are given approximately by

$$
\lambda_{1,2}=\frac{-b_{L} \pm \sqrt{b_{L}^{2}-4 a_{L} c_{L}}}{2 a_{L}}
$$

$$
\lambda_{3,4}=\frac{-d_{L} \pm \sqrt{d_{L}^{2}-4 c_{L} e_{L}}}{2 c_{L}}
$$

The eigenvalues $\lambda_{1,2}$ correspond to the pitch mode (fast oscillation of the kite about point $Q$ ), and $\lambda_{3,4}$ describe the pendular mode (slow oscillation of the main line about the ground attachment point).

A numerical survey of coefficients $a_{L}-e_{L}$ shows that, within the range of parameters of interest, $b_{L}^{2}<4 a_{L} c_{L}$ and $b_{L}>0$; since $b_{L} / 2 a_{L}>0$, the pitch mode is a stable oscillatory mode. For the pendular mode, we numerically found the conditions $d_{L}^{2}<4 c_{L} e_{L}$ and $c_{L}>0$. Therefore, the pendular mode is oscillatory, and its stability boundary is given by the condition $d_{L}=0$ or

$$
\begin{aligned}
& \left(\epsilon_{c} \tilde{x}_{0}-\epsilon_{l} c \delta\right) \theta^{*} s\left(\Gamma^{*}+\theta^{*}\right)\left(2 \theta^{*} s \Gamma^{*}-c \Gamma^{*}\right) \\
& \quad+2 \epsilon_{c} \tilde{x}_{\alpha} \theta^{* 2}\left[s \theta^{*}-\theta^{*} s \Gamma^{*} s\left(\Gamma^{*}+\theta^{*}\right)\right]- \\
& \quad \frac{\epsilon_{l}}{\sigma} s\left(\delta-\theta^{*}\right) c\left(\Gamma^{*}+\theta^{*}\right)\left(c \Gamma^{*}-2 \theta^{*} s \Gamma^{*}\right)=0
\end{aligned}
$$

in which we used Eqs. (A6-A8), and ignored high-order terms in $\epsilon_{l}$, $\epsilon_{c}$, and $\epsilon_{\mathrm{y}}$.

Equation (24) is a useful analytical condition for kite design. It provides a relationship between the relative position of point $Q$, the center of mass, and the center of pressure of the kite (controlled by $\epsilon_{l}$, $\epsilon_{c}$, and $\left.\tilde{x}_{0}\right)$ with aerodynamic and gravity forces $\left(\tilde{x}_{\alpha}\right.$ and $\left.\sigma\right)$. It does not depend on the moment of the inertia $I_{y}$ (or $\epsilon_{y}$ ) because the instability consists of a motion of the main line, and the oscillation of the kite about point $Q$ does not play an important role. We remark that Eq. (24) can be applied not only to kites, but also to any aircraft towed to a line much larger that its characteristic dimension.

Figure 2 shows the longitudinal stability of the equilibrium position in the $W_{0}-\delta$ plane. Both the Jacobian of system (20) at the equilibrium state and its eigenvalues were computed numerically, and stable (unstable) states were plotted with black (gray) color. As

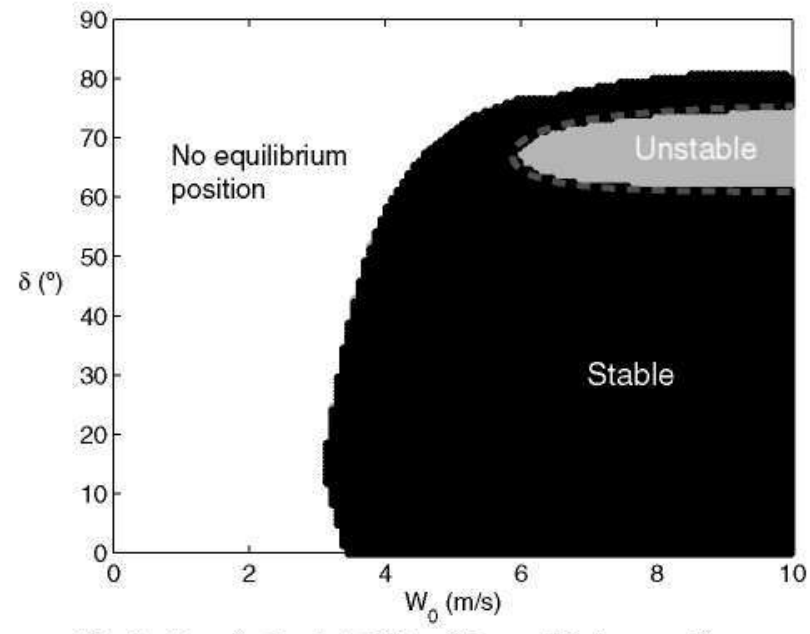

Fig. 2 Longitudinal stability of the equilibrium position. 
shown in [9], a supercritical Hopf bifurcation happens at the boundary stability. The solutions of Eq. (24), dashed line in Fig. 2, perfectly fit this boundary.

\section{Lateral Stability}

The lateral-directional perturbations $\boldsymbol{x}_{D}^{1} \equiv\left[\begin{array}{lll}\varphi^{1} & \psi^{1} & \phi^{1}\end{array}\right]$ are governed by

$$
M_{D}^{*} \ddot{x}_{D}^{1}+D_{L}^{*} \dot{x}_{D}^{1}+K_{D}^{*} x_{D}^{1}=0
$$

in which $M_{D}^{*}, D_{D}^{*}$, and $K_{D}^{*}$ are given by Eqs. (A10-A 12 ). Following a procedure similar to Sec. III.B, one finds the characteristic polynomial:

$$
a_{D} \lambda^{6}+b_{D} \lambda^{5}+c_{D} \lambda^{4}+d_{D} \lambda^{3}+e_{D} \lambda^{2}+f_{D} \lambda+g_{D}=0
$$

with coefficients $a_{D}-g_{D}$ cumbersome functions of the parameters.

The number of real and complex solutions of Eq. (26) depends on the parameters $W_{0}$ and $\delta$. For instance, at $W_{0}=8 \mathrm{~m} / \mathrm{s}$, the real and imaginary parts of the eigenvalues vs the parameter $\delta$ are shown in Figs. 3 and 4 . The eigenvalues have been normalized with their respective values at $\delta=0$ to help the visualization. (See the reference values in the caption.) Modes called 1 and 2 are real in the full $\delta$ range analyzed. Their eigenvectors show that mode 1 corresponds to a roll motion of the kite with no excitation of the main line, and mode 2 involves a yaw motion coupled with a lateral displacement of the main line. The eigenvalues have been normalized with their

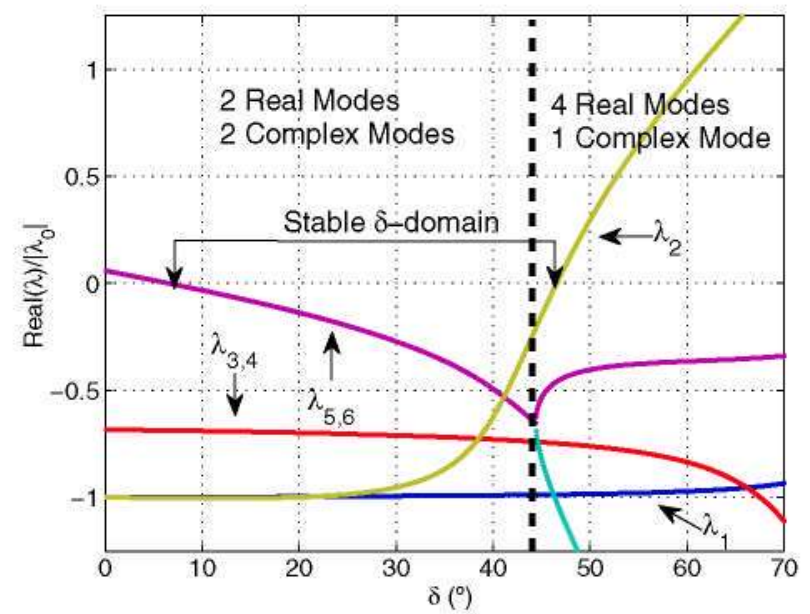

Fig. 3 Normalized real part of the lateral-directional eigenvalues.

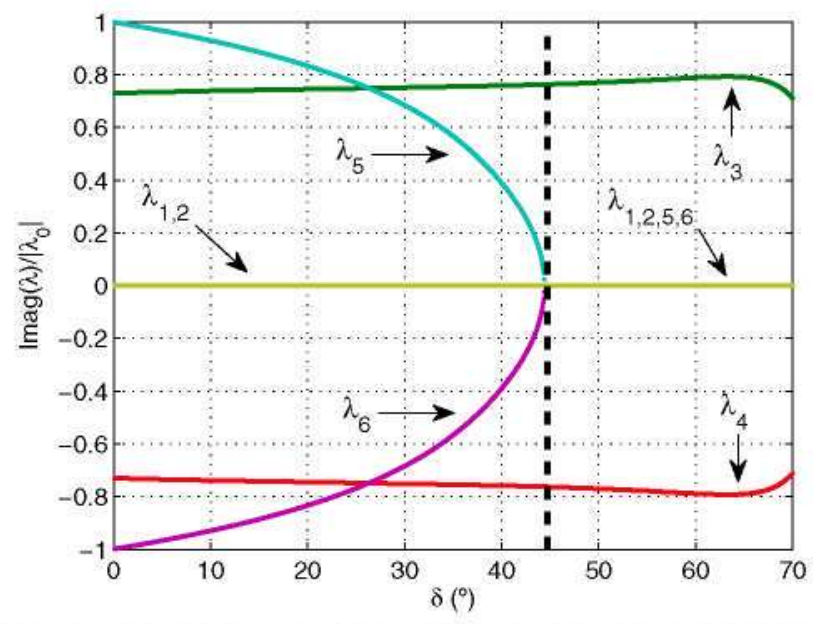

Fig. 4 Normalized imaginary part of the lateral-directional eigenvalues. respective values at $\delta=0\left(|\lambda|_{0}\right)$ to help the visualization. The references values are $\left|\lambda_{0}\right|_{0}=279.9,\left|\lambda_{2}\right|_{0}=0.43,\left|\lambda_{3,4}\right|_{0}=12.84$, and $\left|\lambda_{5,6}\right|_{0}=1.58$. Modes 1 and 2 are unstable if $\delta<6.3 \mathrm{deg}$ and $\delta>46.6 \mathrm{deg}$, respectively. The eigenvalues 3 and 4 are a pair of complex conjugated solutions, and their eigenvectors indicate a yaw motion with no excitation of the main line. Finally, the eigenvalues 5 and 6 appear as a pair of complex conjugated solutions for $\delta<44.5$ and as two real solutions for $\delta>44.5$. Both kite yaw motion and main-line displacement are involved in modes 5 and 6 . As indicated in Fig. 3, the equilibrium state of the kite is stable within the range $6.3 \mathrm{deg}<\delta<46.6 \mathrm{deg}$.

Figure 5 shows the lateral stability of the equilibrium state in the $W_{0}-\delta$ plane computed numerically from Eq. (26). Parameter values giving rise to an eigenvalue with positive real part (unstable behavior) are plotted with gray color. Clearly, the angle $\delta$ that controls the bridle geometry should be below a certain threshold to have a stable behavior. For high wind velocity and low $\delta$, there is a second unstable parametric region. The two stability boundaries shown in Fig. 5 can be derived from Eq. (26). Using the Routh-Hurwitz stability criterion, we found that the upper stability boundary is given by the condition $g_{D}=0$, which reads

$$
C_{z \alpha} \theta^{*} v f_{x}+\frac{\epsilon_{l}}{\mu \tilde{V}_{0}^{2}} u s \delta=0
$$

in which functions $f_{x}, u$, and $v$ are given by Eqs. (A9), (A13), and (A14), respectively. The Routh-Hurwitz criterion also shows that the lower stability boundary in Fig. 5 is described by the condition $f_{D}\left(d_{D} e_{D}-c_{D} f_{D}\right)-d_{D}^{2} g_{D}=0$, which is a complex function of the parameters.

As shown in Fig. 3, the upper stability boundary happens because the eigenvalue $\lambda_{2}$, which is real, changes its sign. A numerical integration of system (15), with parameter values in the unstable domain but close to the upper boundary, and with initial condition at the fixed point perturbed by the unstable eigenvector, shows a lateral kite motion until it reaches the ground. This simple numerical test suggests that probably there is a subcritical pitchfork bifurcation at the upper boundary. On the other hand, the real part of the complex conjugated eigenvalues $\lambda_{5,6}$ changes its sign at the low stability boundary of Fig. 5. A numerical integration of the equations, similarly to the previously described, but with parameter values in the lower unstable domain, gives rise to a lateral oscillation of the kite with increasing amplitude until it touches the ground. A subcritical Hopf bifurcation may happen at the lower boundary.

\section{Kite Dynamics Under Nonsteady Wind Conditions}

For nonsteady wind conditions, the equilibrium state of the kite is destroyed, and a more complex dynamics appears. Here, we assume

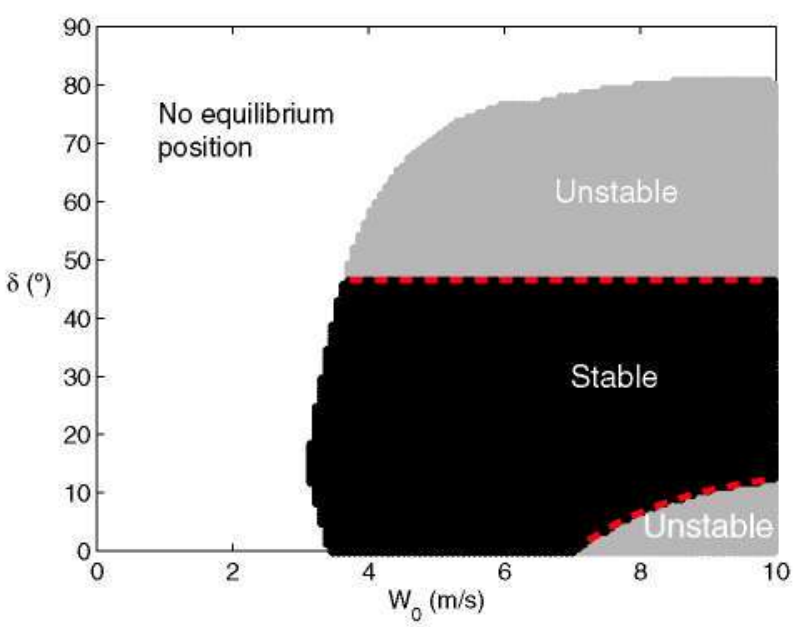

Fig. 5 Lateral-directional stability of the equilibrium position. 


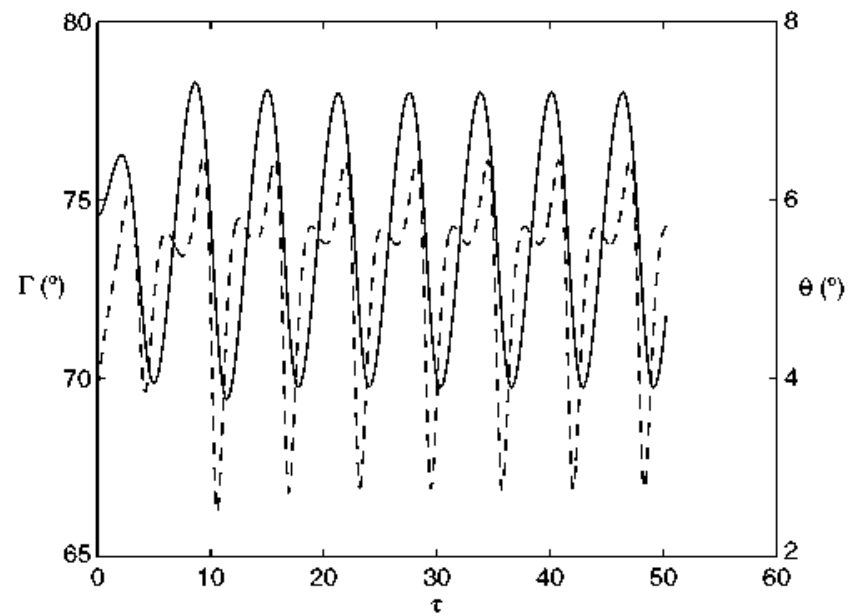

Fig. 6 Evolution of the angles $\theta$ (dashed) and $\Gamma$ (solid).

the model given by Eq. (12), that is, a constant wind velocity $w_{0}=$ $8 \mathrm{~m} / \mathrm{s}$ perturbed by a sinusoidal variation of amplitude $\tilde{V}_{1}=0.15$ and normalized frequency $\tilde{\Omega}=1$. For simplicity, we restrict the analysis to the longitudinal motion of the kite and set $\varphi=\psi=$ $\phi=0$. A numerical integration of system (15), started with an initial condition equal to the steady state of the kite with a constant wind velocity, indicates that the kite evolves toward a periodic orbit with (normalized) period equal to $2 \pi / \tilde{\Omega}$. As shown in Fig. 6, after a short transient, both the pitch angle $\theta$ and the elevation angle of the main line $\Gamma$ converge to a periodic orbit.

Removing the transient by a direct integration of the equations, however, is not an efficient method to analyze periodic orbits. A better procedure is the predictor-corrector method described in [17], which computes a branch of periodic orbit as a parameter is varied together with the eigenvalues of the monodromy matrix (an indicator of the linear stability of the orbit). Figure 7 shows a bifurcation diagram of a branch of periodic orbits; the maximum values of the pitch angle $\theta_{\max }$ and the line elevation angle $\Gamma_{\max }$ reached along the periodic orbit are plotted vs the control parameter $\tilde{\Omega}$. An analysis of the Floquet multipliers (not shown) indicates that the full branch is stable because all the eigenvalues of the monodromy matrix have moduli lower than 1.

Figure 7 also displays an interesting resonance phenomenon. The amplitudes of the periodic orbits are higher if the frequency that modulates the wind-velocity perturbation is close to an eigenfrequency of the kite; from Eq. (21), one finds $\lambda_{\text {pendular }}=$ $-0.2693-0.6997 i$ and $\lambda_{\text {pitch }}=-20.6619+35.7961 i$, whose

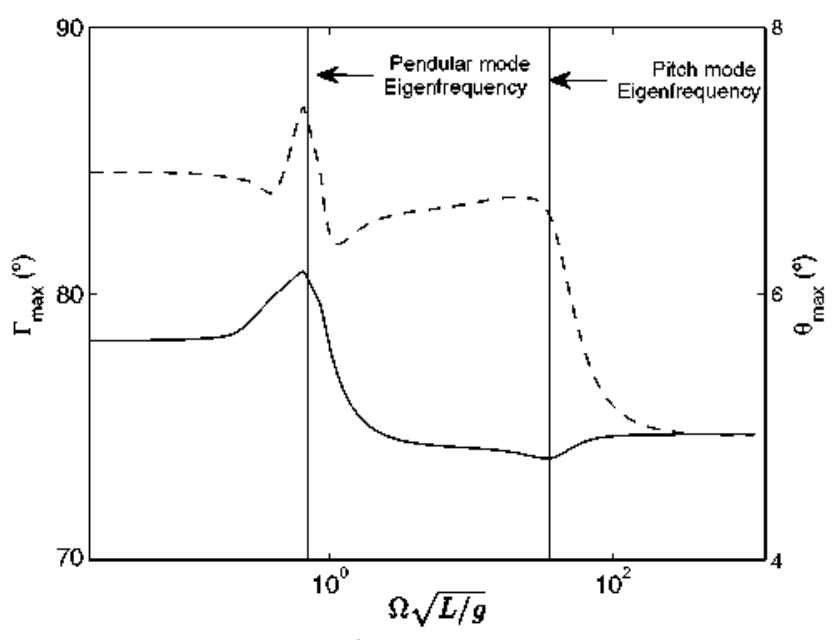

frequencies are plotted with a black line in Fig. 7. Clearly, the kite response is stronger if the wind velocity has a Fourier component close to one of the natural frequencies of the system.

\section{Conclusions}

A simple model to study both the longitudinal and lateraldirectional dynamics of a kite under steady and nonsteady windvelocity conditions has been described. Its five degrees of freedom, including two angles, $\Gamma$ and $\varphi$, that describe the state of the main line, and three Euler angles, $\theta, \psi$, and $\phi$, capture the most important features of the kitelike dynamics. Future works, however, may consider a flexible main line, an effect here ignored, but probably not negligible for long lines.

Under steady wind conditions, the equilibrium state of the kite can be unstable for certain combinations of wind velocities $W_{0}$ and bridle angles $\delta$ (see Figs. 2 and 5). This work has presented two analytical relationships among the kite parameters that must be satisfied to design longitudinal and lateral stable kites. Because the current model does not include aerodynamic force along the $x_{B}$ direction. both relations are conservative. (Preliminary calculations, including such a force, indicate an enhancement of the kite stability.) The longitudinal eigenvalue that loses stability is imaginary and a branch of stable periodic orbits born at the supercritical Hopf bifurcation [9]. On the other hand, the lateral eigenvalues that become unstable can be real or complex. Probably, a subcritical pitchfork bifurcation and a subcritical Hopf bifurcation happen at the upper and lower stability boundaries of Fig. 5 .

For nonsteady wind conditions, the kite has no equilibrium states. but stable periodic orbits. Using a predictor-corrector algorithm, this work has illustrated a resonance phenomenon involving the natural frequencies of the kite (both pitch and pendular longitudinal eigenfrequencies) and the frequency of the perturbed wind velocity. If the frequency of the wind, which acts as a forcing term. is close to a natural frequency of the kite, then a high-amplitude periodic orbit happens. In real conditions, the wind presents a broad spectrum of frequencies instead of an isolated frequency. If a calm flight of the kite is desired (for instance, to take photography), then the kite, bridle, and main line may be designed to avoid natural eigenfrequencies close to the dominant frequencies of the wind.

The tensor notation and Lagrangian formulation introduced here give rise to a compact model free of constraint forces. These properties make it suitable for the incorporation of new effects. In particular, a model for kites with time-dependent bridle geometry can be implemented. The performances of this model as a wind-power system operating in pumping mode will be presented in a forthcoming work.

\section{Appendix: Auxiliary Calculations}

The rotation matrix $\boldsymbol{R}$ that relates the Earth and body frames is

$$
\boldsymbol{R}=\left(\begin{array}{ccc}
c \psi c \theta & s \psi c \theta & -s \theta \\
c \psi s \theta s \phi-s \psi c \phi & s \psi s \theta s \phi+c \psi c \phi & c \theta s \phi \\
c \psi s \theta c \phi+s \psi s \phi & s \psi s \theta c \phi-c \psi s \phi & c \theta c \phi
\end{array}\right)
$$

The angular velocity of the kite and the velocity of its center of mass $G$ are written as $\omega=S^{(\omega)} \dot{x}$ and $V_{G}=S^{(v)} \cdot \dot{x}$, in which $S^{(\omega)}$ and $S^{(1)}$ are given by

$$
\boldsymbol{S}^{(\omega)}=\left(\begin{array}{ccccc}
0 & 0 & 0 & -s \theta & 1 \\
0 & c \phi & 0 & c \theta s \phi & 0 \\
0 & -s \phi & 0 & c \theta c \phi & 0
\end{array}\right)
$$




$$
\boldsymbol{S}^{(t)}=\left[\begin{array}{ccccc}
s \Gamma c \varphi & \epsilon_{l} c \psi f_{2} & c \Gamma s \varphi & \epsilon_{l}\left(s \psi f_{1}-s \delta c \psi s \phi\right) & \epsilon_{l} s \delta(c \psi s \theta s \phi-s \psi c \phi) \\
s \Gamma s \varphi & \epsilon_{l} s \psi f_{2} & -c \Gamma c \varphi & -\epsilon_{l}\left(c \psi f_{1}+s \delta s \psi s \phi\right) & \epsilon_{l} s \delta(s \psi s \theta s \phi+c \psi c \phi) \\
-c \Gamma & \epsilon_{l} f_{1} & 0 & 0 & \epsilon_{l} s \delta c \theta s \phi
\end{array}\right]
$$

From Eqs. (2) and (4), and $V_{G}=S^{(j)} \cdot \dot{x}$, one finds the matrix $M \equiv M_{L}+M_{R}$ appearing in Eq. (14). One finds

$$
\begin{gathered}
M_{L}=\left\{\begin{array}{ccccc}
1 & \epsilon_{l}\left(s \Gamma c \nu f_{2}-c \Gamma f_{1}\right) & 0 & -\epsilon_{l} s \Gamma\left(s \delta c \nu s \phi+s \nu f_{1}\right) & \epsilon_{l} s \delta[s \Gamma(c \nu s \theta s \phi+s \nu c \phi)-c \Gamma c \theta s \phi] \\
- & \epsilon_{l}^{2}\left(1-s^{2} \delta s^{2} \phi\right) & \epsilon_{l} c \Gamma s \nu f_{2} & -\epsilon_{l}^{2} s \delta s \phi f_{2} & \epsilon_{l}^{2} s \delta c \delta s \phi \\
- & - & c^{2} \Gamma & -\epsilon_{f} c \Gamma\left(s \delta s \nu s \phi-c \nu f_{1}\right) & \epsilon_{l} s \delta c \Gamma(s \nu s \theta s \phi-c \nu c \phi) \\
- & - & - & \epsilon_{f}^{2}\left(c^{2} \theta s^{2} \phi+f_{3}^{2}\right) & -\epsilon_{l}^{2} s \delta f_{3} \\
- & - & - & - & \epsilon_{l}^{2} s^{2} \delta
\end{array}\right\} \\
M_{R}=\left[\begin{array}{ccccc}
0 & 0 & 0 & 0 & 0 \\
- & \epsilon_{y}^{2} c^{2} \phi+\epsilon_{z}^{2} s^{2} \phi & 0 & \frac{1}{2}\left(\epsilon_{y}^{2}-\epsilon_{\bar{v}}^{2}\right) c \theta s 2 \phi & 0 \\
- & - & 0 & 0 & 0 \\
- & - & - & \epsilon_{x}^{2} s^{2} \theta+c^{2} \theta\left(\epsilon_{z}^{2} c^{2} \phi+\epsilon_{y}^{2} s^{2} \phi\right) & -\epsilon_{x}^{2} s \theta \\
- & - & - & - & \epsilon_{x}^{2}
\end{array}\right]
\end{gathered}
$$

in which we introduced the angle $\nu \equiv \varphi-\psi$ and the auxiliary function $f_{3}=\sin \delta \sin \theta+\cos \delta \cos \theta \cos \phi$.

The matrices $\boldsymbol{M}_{L}, D_{L}$, and $\boldsymbol{K}_{L}$ in Eq. (20) are given by

$$
\begin{gathered}
\boldsymbol{M}_{L}=\left[\begin{array}{cc}
1 & -\epsilon_{l} c(\delta-\Gamma-\theta) \\
- & \epsilon_{f}^{2}+\epsilon_{y}^{2}
\end{array}\right] \\
\frac{\boldsymbol{D}_{L}}{\mu \tilde{V}_{0} C_{z \alpha}}=\left(\begin{array}{cc}
-c(\Gamma+\theta)(c \Gamma-2 \theta s \Gamma) & \epsilon_{l} c(\Gamma+\theta)[c(\delta-\theta)-2 \theta s(\delta-\theta)] \\
f_{x}(2 \theta s \Gamma-c \Gamma)+\epsilon_{c} \hat{x}_{a} \theta c \Gamma & \epsilon_{l}\left\{f_{x}[c(\delta-\theta)-2 \theta s(\delta-\theta)]-\epsilon_{c} \tilde{x}_{\alpha} \theta c(\delta-\theta)\right\}
\end{array}\right) \\
\boldsymbol{K}_{L}=\left\{\begin{array}{cc}
-s \Gamma-\mu \tilde{V}_{0}^{2} s(\Gamma+\theta) C_{-\alpha} \theta & \mu \tilde{V}_{0}^{2} C_{z a}[c(\Gamma+\theta)-\theta s(\Gamma+\theta)] \\
0 & \mu \tilde{V}_{0}^{2} C_{z \alpha}\left[\epsilon_{c}\left(\tilde{x}_{0}-2 \tilde{x}_{\alpha} \theta\right)-\epsilon_{l} c \delta\right]-\epsilon_{l} s(\delta-\theta)
\end{array}\right\}
\end{gathered}
$$

in which

$$
f_{x} \equiv \epsilon_{c}\left(\tilde{x}_{0}-\tilde{x}_{a} \theta\right)-\epsilon_{f} c \delta
$$

On the other hand, matrices $\boldsymbol{M}_{D}, \boldsymbol{D}_{D}$, and $\boldsymbol{K}_{D}$ in $\mathrm{Eq}$. (25) are

$$
\begin{gathered}
M_{D}=\left[\begin{array}{ccc}
c^{2} \Gamma & \epsilon_{l} c(\delta-\theta) c \Gamma & -\epsilon_{l} s \delta c \Gamma \\
- & \epsilon_{\bar{l}}^{2} c^{2}(\delta-\theta)+\epsilon_{x}^{2} s^{2} \theta+\epsilon_{z}^{2} c^{2} \theta & -\epsilon_{\bar{l}}^{2} s \delta c(\delta-\theta)-\epsilon_{x}^{2} s \theta \\
- & - & \epsilon_{l}^{2} s^{2} \delta+\epsilon_{\bar{x}}^{2}
\end{array}\right] \\
\frac{D_{D}}{\mu \tilde{V}_{0}}=\left\{\begin{array}{ccc}
-C_{Y \beta} c^{2} \Gamma & -\epsilon_{l} C_{Y \beta} c(\delta-\theta) c \Gamma & \epsilon_{l} C_{Y j} s \delta c \Gamma \\
u \cos \Gamma & \epsilon_{l} c(\delta-\theta) u-\epsilon_{b} \tilde{V}_{0}\left[C_{l \hat{p}} s^{2} \theta+C_{n \tilde{r}} c^{2} \theta\right] & -\epsilon_{l} u s \delta+\epsilon_{b} C_{l \dot{p}} \tilde{V}_{0} s \theta \\
v c \Gamma & \epsilon_{l} v c(\delta-\theta)+\epsilon_{b} C_{l \bar{p}} \tilde{V}_{0} s \theta & -\left(\epsilon_{f} v s \delta+\epsilon_{b} C_{l \bar{p}} \tilde{V}_{0}\right)
\end{array}\right\}
\end{gathered}
$$

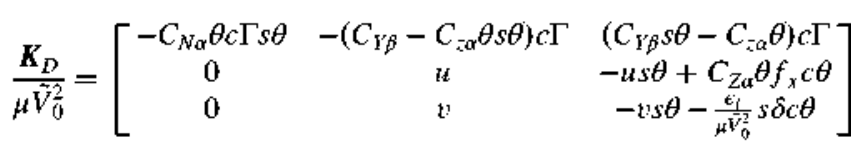

with

$$
\begin{gathered}
u=\epsilon_{b}\left(C_{n \beta} c \theta-C_{\mid \beta} s \theta\right)-\epsilon_{t} C_{Y \beta} c(\delta-\theta) \\
v=\epsilon_{l} C_{Y \beta} s \delta+\epsilon_{b} C_{\mid \beta}
\end{gathered}
$$

We remark that $\boldsymbol{M}_{L}, \boldsymbol{D}_{L}$, and $\boldsymbol{K}_{L}$ in Eq. (20) and $\boldsymbol{M}_{D}, \boldsymbol{D}_{D}$, and $\boldsymbol{K}_{D}$ in Eq. (25) must be evaluated at the equilibrium position $x^{*}$ given by $\mathrm{Eq} .(16)$.

\section{References}

[1] Schmehl, R., "Kiting for Wind Power," Wind Systems, Vol. 27. No. 7. 2012, pp. 36-43.

[2] Gagiano. M. C. L., Milanese, M.. and Ippolito, M.. "KiteGen Project: Control as Key Technology for a Quantum Leap in Wind Energy Generators," Proceedings of Anerican Control Conference, July 2007, pp. 3522-3528, doi: 10.1 109/ACC. 2007.4282697

[3] Loyd, M. L.. "Crosswind Kite Power," Joumal of Energy, Vol. 4, No. 3. 1980, pp. 80-4075.

[4] Ockels, W. J., "Laddermill, a Novel Concept to Exploit the Energy in the Airspace," Aircruft Design, Vol. 4, No. 2. 2001, pp. 81-97. doi: 10.1016/S1369-8869(01)00002-7

[5] Lansdorp, B., and Ockels. W. J., "Comparison of Concepts for HighAltitude Wind Energy Generation with Ground Based Generator:" China International Renerable Energy Equipment and Technology Exhibition and Conference, Beijing, May 2005. 
[6] Bairstow. L.. "The Stability of Kite Ballons: Mathematical Investigation," RM 208, Aeronautical Research Council, U.K., Dec. 1915

[7] Glauert, H.. "The Stability of a Body Towed by a Light Wire."RM 1312. Aeronautical Research Council. U.K., 1930.

[8] Adomaitis, R. A., "Kites and Bifurcation Theory:" SIAM Review, Vol. 31, No. 3. 1989. pp. 478-483. doi: $10.1137 / 1031092$

[9] Sánchez-Arriaga, G. "Dynamics and Control of Single Line Kites." Aeronautical Joumal, Vol. 110, No. 1111, 2006, pp. 615-621.

[10] Terink. E., Breukels. J., Schmehl, R., and Ockels. W., "Flight Dynamics and Stability of a Tethered Inflatable Kiteplane." Joumal of Aircraft, Vol. 48, No. 2. 2012. pp. 503-513. doi: $10.2514 / 1.0031108$

[11] Alexander, K., and Stevenson, J., "Kite Equilibrium and Bridle Length," Aeronautical Joumal, Vol. 105, No. 1051, 2001, pp. 535-541.

[12] Veldman. S., Bersee. H., Vermeeren. C., and Bergsma, O., "Conceptual Design of a High Altitude Kite," 43 rd AIAA/ASME/ASCE/AHS/ASC Strtictures, Strtitural Dynamics, and Materials Conference. ALAA Paper 2002-1735, 2002. doi: $10.2514 / 6.2002-1735$

[13] de Groot, S. G. C., Breukels, J., Schmehl, R., and Ockels, W., "Modeling Kite Flight Dynamics Using a Multibody Reduction Approach."
Joumal of Guidance, Control, and Dynamics. Vol. 34. No, 6, 2011. pp. $1671-1682$.

doi: $10.2514 / 1.52686$

[14] Diehl, M., Magni. L., and Nicolao, G.. "Efficient NMPC of Unstable Periodic Systems Using Approximate Infinite Horizon Closed Loop Costing," Annual Review's in Control, Vol. 28, No. 1, 2004, pp. $37-45$.

doi: $10.1016 \mathrm{j}$.arcontrol.2004.01.011

[15] Williams, P., Lansdorp, B., and Ockels, W., "Nonlinear Control and Estimation of a Tethered Kite in Changing Wind Conditions," Journal of Guidance, Control, and Dynamics, Vol. 31. No. 3, 2008. pp. 793-799. doi:10.2514/1.31604

[16] Williams, P.. Lansdon. B., and Ockels, W. "Optimal Crosswind Towing and Power Generation with Tethered Kites," Joumal of Guidance, Control, and Dvnanics, Vol. 31. No. 1, 2008, pp. 81-93. doi: $10.2514 / 1.30089$

[17] Lara. M.. and Peláez, J.. "On the Numerical Continuation of Periodic Orbits: An Intrinsic, 3-Dimensional, Differential, Predictor-Corrector Algorithm," Astronomy and Astrophysics. Vol. 389, No. 2, July 2002. pp. 692-701.

doi: $10.1051 / 0004-6361: 20020598$ 